\title{
A Discussion of the Favorable Factors for the Economic System's Utility Growth Rate
}

\author{
Lunhan Luo and Jianan Fang \\ School of Information Science and Technology, Donghua University, Shanghai 201620, China \\ Correspondence should be addressed to Jianan Fang; jafang@dhu.edu.cn
}

Received 2 August 2013; Revised 26 September 2013; Accepted 23 October 2013

Academic Editor: Yang Tang

Copyright ( 2013 L. Luo and J. Fang. This is an open access article distributed under the Creative Commons Attribution License, which permits unrestricted use, distribution, and reproduction in any medium, provided the original work is properly cited.

\begin{abstract}
Some virtual economic systems are constructed. The agents in the systems are linked crossover by different network structures and endowed with goods and the same symmetric Cobb-Douglas utility function. Their Cobb-Douglas utility growth rates (UGR) are compared with each other after the systems reach their eventual equilibriums. We try to discover how the network structures and how the endowments affect the UGR. Furthermore, the possible factors including group size, trade sequence, turnover, and number of trades are taken into account. Some but not all of the factors listed above may impact the UGR. The aim of this paper is to select the effective ones and try to improve the UGR by manipulating these factors. We discovered that the small-world network surpasses other networks to achieve the highest UGR. Besides, another way to improve UGR is to endow the agents with proper volume of goods.
\end{abstract}

\section{Introduction}

In the past few years, the discovery of small-world network has stimulated a large interest in its characteristics from many principles [1-4]. Consciously or unconsciously, we are relying on and contributing to the small-world network. In the 1990s, Watts and Strogatz [5] have shown that the connection topology of some biological, technological, and social networks is neither completely regular nor completely random [6] but stays somehow in between these two extreme cases [7]. They have defined the model of this network organization as small-world network. The discovery of smallworld network has triggered a large interest from many principles in the study of its properties. Researchers have focused their attention on different aspects: Internet $[8,9]$, epidemic $[10,11]$, social networks $[12,13]$, and geography [14$16]$.

Scientists have studied the economic system through the dynamics of networks. Bell [17] investigated a variety of trading structures focusing on the speed of convergence to an equilibrium price as well as the persistence of neighborhood effects. As the number of trades is a measure of the speed of convergence, the author found that more centralized networks, compared to more sparse networks, converge with fewer trades and have less residual price variation.

Li et al. [18] proposed some factors and principles influencing the evolutionary complexity of complex adaptive supply networks (CASNs). The external environmental factors, such as government regulation, market demand, and market structure, were considered to be the dominant forces that shape the gradual evolution of networks. They appeared to have a long-term impact on the evolution. However, a firm's strategies, product structure, technology, and organization appeared to be the internal factors that exert an immediate influence on the evolution of CASNs. Among these factors, costs and quality considerations appeared to be the primary forces that influence the structure complexity, centralization, and formalization of CASNs.

Wilhite [19] compared the search and negotiation costs of different network structures and their mechanisms to initiate and proceed a trade. Small-world network has been proven to have several characteristics of general interest in economics. First, all local trades can economize on these costs. Second, when a few overlapping traders belonging to other trade groups exist, goods can be traded between any pairs of agents with relatively few exchanges. Accordingly, 
it has been revealed that small-world networks have the ability to strike a balance between the advantages and costs of having many trade partners.

It has been proven that the small-world feature is an underlying constitutive property of many natural and artificial networks [20-22]. We believe that it is not an occasional incident. The principle of survival of the fittest gives us the inspiration that the small-world feature may be rewarded for its advantages when constructing an efficient but low-cost network. However, it is hard to define advantages without a specific environment. Thus, we place the analysis of smallworld feature in a data-driven economic system. To capture a clearer result, several network features under virtually identical circumstances are compared to see the difference.

The structure of the paper is designed as follows. Section 2 introduces the economy model and the relevant complex network theory. Based on the model and theory, Section 3 designs the experiment plans. In Section 4, we identify some factors which may affect UGR and try to improve the UGR by manipulating them. In Section 5 , the main contributions of this study are summarized.

\section{Economy Model and Network Theory}

In this section, an economy model which is derived from the one proposed by Wilhite [19] is introduced to define the transaction process. For a better understanding of the network structures in this paper, a brief description of the network statistic characteristics is provided.

2.1. Economy Model. In this model, a determined amount of independent agents is created. Two types of goods, one of which must be traded in whole units and the other is infinitely divisible, are assigned to each agent as the existing wealth to circulate in the market. The portion of the goods is randomly assigned at the beginning of the experiment. There is no production and no imports, thus the aggregate stock of goods at the beginning of the experiment is the stock at the end.

Each agent's objective is to improve its own CobbDouglas utility function [23] in each period by engaging in voluntary trade. Formally, $U^{i}$ depends on the individual's existing wealth of $g_{1}$ and $g_{2}$. Consider the following:

$$
U^{i}=g_{1}^{i} g_{2}^{i}, \quad i \in\{1, \ldots, n\},
$$

where $n$ is the amount of agents.

The entire economic society is composed of every transaction. A transaction is a process in which two nodes exchange the goods since one node's questing for a negotiating price chance is being responded. An opportunity for mutually beneficial transaction exists if the marginal rates of substitution (mrs) of two agents differ. mrs reflect the agent's willing to give up $g_{2}$ in exchange for a unit of $g_{1}$. With the utility function in (1), the mrs of agent $i$ are

$$
\mathrm{mrs}^{i}=\frac{U^{\prime}\left(g_{1}^{i}\right)}{U^{\prime}\left(g_{2}^{i}\right)}=\frac{g_{2}^{i}}{g_{1}^{i}}, \quad i \in\{1, \ldots, n\},
$$

where $U^{\prime}(\cdot)$ is the first derivative of $U$.
The model assumes that each agent reveals its mrs. In the experiment, agents search for beneficial trade opportunities according to the mrs and then establish a price to initiate a transaction. Any agent can either trade $g_{2}$ for $g_{1}$ or trade $g_{1}$ for $g_{2}$ at the expense of price. Throughout these experiments, the trading price $p_{i, j}$ between agent $i$ and agent $j$ is set according to the following rule:

$$
p_{i, j}=\frac{g_{2}^{i}+g_{2}^{j}}{g_{1}^{i}+g_{1}^{j}}, \quad i, j \in\{1, \ldots, n\} .
$$

The questing node would pay $p_{i, j}$ by $g_{2}$ to exchange one unit of $g_{1}$. Meanwhile, the responding node would add $p_{i, j}$ to its stock of $g_{2}$ and sell one unit of $g_{1}$ to the questing node. The transaction proceeds as long as the trade benefits each node's $U^{i}$ and stops when one of the nodes is lacking $g_{1}$ or cannot afford the price $p_{i, j}$. Each transaction is atomic in the experiments; namely, it would not be suspended till the whole process is fulfilled. In the experiments, every active transaction will be considered as one time trade but two trade volumes, since the income and outcome strings are both taken into account.

Once a transaction stops, the questing node will search again for a new opportunity according to the trade rules until no node responds to it, and so on. Another node is selected as questing node to engage in a transaction. The economy society evolves like this and stops at the network's equilibrium.

Equilibrium is a point when agents cannot find trading opportunities that benefit any individuals. Feldman [24] studied the equilibrium characteristics of welfare-improving bilateral trade and showed that as long as all agents possess some nonzero amount of one of the commodities (all agents have some $g_{1}$ or all agents have some $g_{2}$ ) then the pairwise optimal allocation is also a Pareto optimal allocation. In this experiment, all agents are initially endowed with a positive amount of both goods, thus the equilibrium is Pareto optimal [25].

2.2. Network Statistic Characteristics. To better understand a network, many network statistic characteristics are significant. The variations of network structure are numerous, but they have common statistical characteristics. The ones we are concerned with the most are listed below.

2.2.1. Degree. In graph theory, the degree of a vertex of a graph is the number of edges incident to the vertex [26]. The maximum degree of a graph and the minimum degree of a graph are the maximum and minimum degrees of its vertices, respectively.

2.2.2. Average Path Length. Average path length (APL) is a concept in network topology defined as the average number of steps along the shortest paths for all possible pairs of network nodes. It measures the efficiency of information or mass transport on a network [27]. Latora and Marchiori [28] defined the cost of a weighted graph as a formula based on the weight and path length. It grows as the population is divided into additional groups. We define the average shortest 


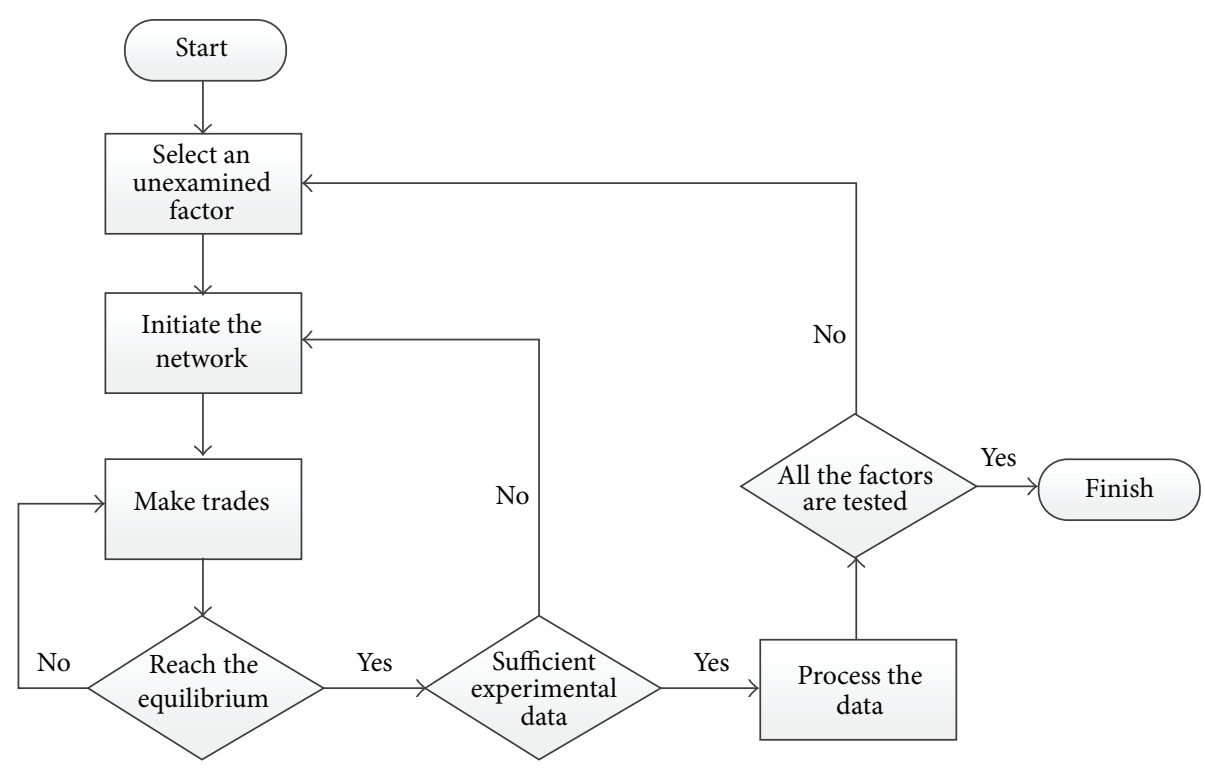

Figure 1: Experimental design.

path as the trade cost to evaluate the network's resource transferring efficiency. We may substitute the term "APL" for "cost" sometimes in the rest of this paper.

2.2.3. Clustering Coefficient. In graph theory, a clustering coefficient measures the degree to which nodes in a graph tend to cluster together (Holland and Leinhardt [29]). In a connected graph, each node has its neighbors. The clustering coefficient of a node is the portion of the actual edges among the nodes' neighbors divided by the maximal possible edges among them, written as

$$
C_{i}=\frac{2\left|\left\{e_{j k}\right\}\right|}{k_{i}\left(k_{i}-1\right)}, \quad e_{j k} \in E, i, j \in\{1, \ldots, n\},
$$

where $k_{i}$ is the neighbors of node $i$ and $e_{j k}$ is the edges among $i$ 's neighbors.

The clustering coefficient for the whole network is given by Watts and Strogatz [5] as the average of the local clustering coefficients of all the vertices $n$ [30]. Consider the following:

$$
\bar{C}=\frac{1}{n} \sum_{i=1}^{n} C_{i}
$$

A larger clustering coefficient value means that the node has a more compact system of connections with its neighbors.

\section{Experimental Design}

This economy model is simulated in a Python program and with repeated simulations to explore the factors that may have an influence on the UGR. The factors being examined in the experiment are
(a) network structures,
(b) group size,

(c) endowments,

(d) trading sequence,

(e) turnover and number of trades.

To begin the experiment, artificial economy society is abstracted from the trade network composed of nodes and edges to conduct the experiment analysis by the network theory. According to the economy model, each society has agents (represented by nodes), trading rules (represented by edges), and goods $g_{1}$ and $g_{2}$ (represented by the endowment of each node).

Then we initiate the experiments. The method we design can discuss any market with nodes in multiple of 10 . More specifically, we raise an instance of 20 nodes network as a case study. Each node is randomly assigned a portion of the society wealth and has the same Cobb-Douglas utility to maximize. Network structures define the edges among the nodes, constraining the extent of trade partners each node can reach.

Then the trade proceeds according to the regime defined by the economy model till the equilibrium is reached. Figure 1 describes the design of the experiment.

We adjust the factors to observe possible variations of UGR. When focusing on a specific factor, we can replicate the initial conditions for each simulation and summarize the equilibrium status under different circumstances. For the reason of studying the factors, we examine peer-to-peer environments; each network is created with the same initial conditions. (1) The group size is 20. (2) The endowments of goods $g_{1}, g_{2}$ are the same. (3) The motivation of each node is to maximize its own Cobb-Douglas utility $U^{i}$. Moreover, the result is a summary of numerous repeated simulations.

3.1. Network Structures. In the experiments, global network, local disconnected network, local connected network, and small-world network are proposed as 4 different trade rules. 


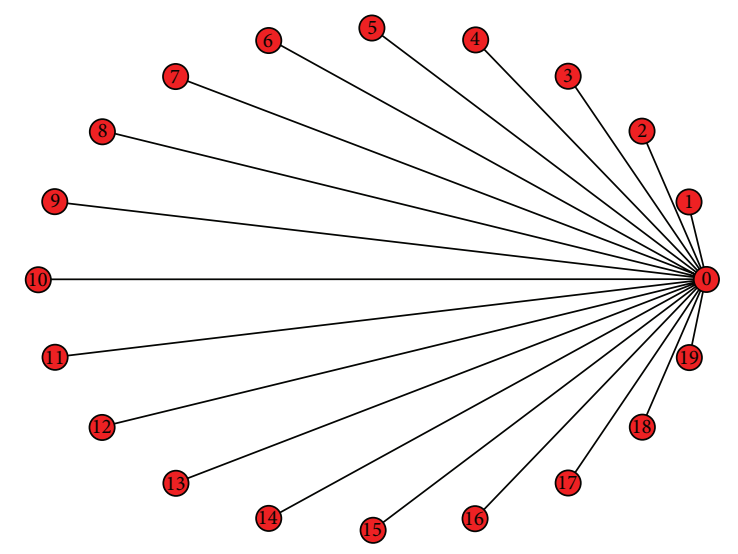

(a) Global network

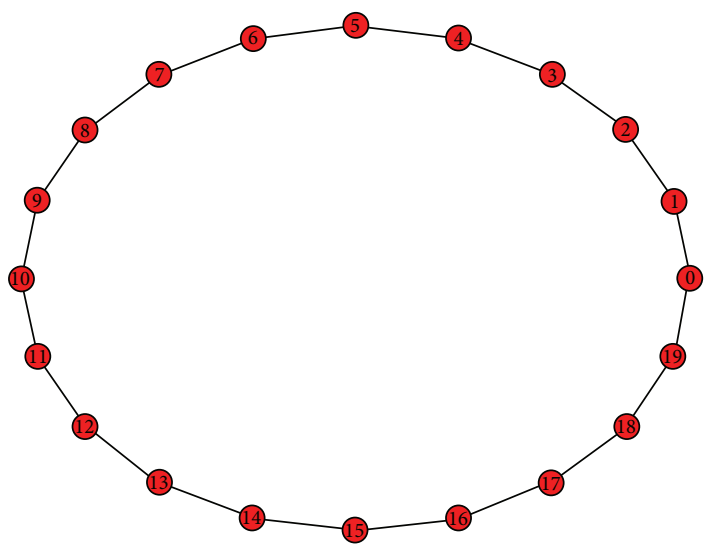

(c) Local connected network

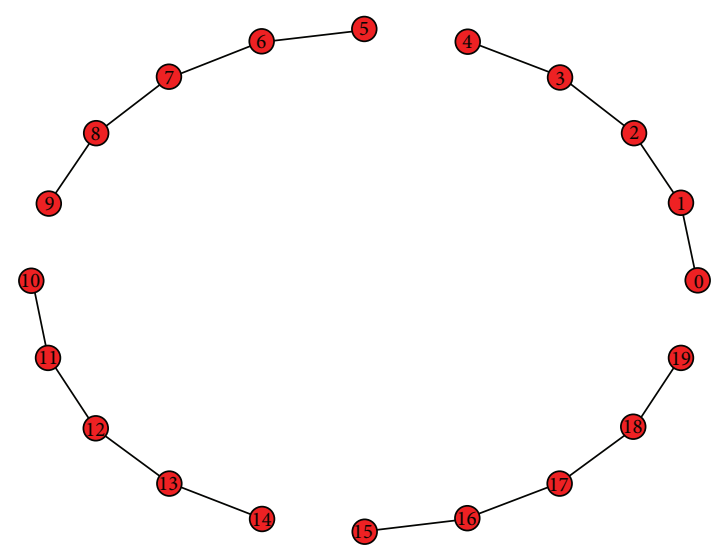

(b) Local disconnected network

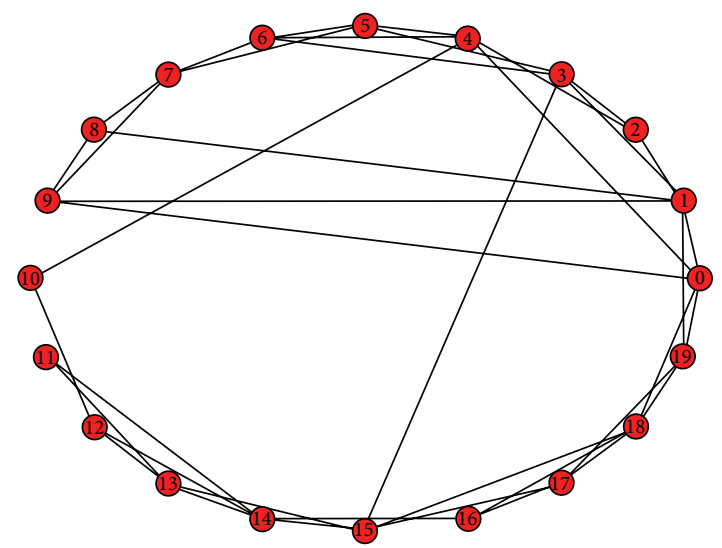

(d) Small-world network

FIGURE 2: Four network structures.

The next step is to compare the dynamic behaviors when these networks reach the equilibriums. We pay particular attention to the influence that may be caused by the network structure. Different experiments are designed to evaluate each factor. Much of the emphasis on networks in the field of economics is about network formation, as described in the work of Jackson and Wolinsky [31] and Ioannides [32]. They construct the models with autonomous agents in which trade networks evolve as agents learn.

Figure 2 shows the sketches of the four networks. The edges among nodes reflect the architectures of trade networks. They act as the trade rules by limiting neighbors each node can reach, contributing to the dynamic mechanism. Throughout these experiments, nodes are not pioneering; that is, they only do direct transaction without further searching for trading opportunities. Thus the extent of potential partners excludes the node with path length 2 or more. Given a defined amount of nodes, if they were in a regular network, every node would gain access to all the other nodes and would have the same number of neighbors and fixed APL. On the contrary, if they were in a random network, all the linking would be unpredictable, and the APL would vary every time. In the real world, most networks' regularity falls in between these extremes. They possess not only much more steady mechanisms worth researching, but also flexibility to raise reformation of system performance.

The structure of global network, comprised of one node selected as hub, and others as common nodes linking to the hub is clear and intuitive. The hub has degree 19 and may gain access to any node with path length 1 , keeping the network on a very low APL since each node is reachable within 2 steps search. Consequently, the global network has a quite small average path length of 1.9. In parallel, all common nodes can trade with the hub.

Despite the centralization of global network, local disconnected network has several independent and structurally similar subnetworks. For experiments's sake, each subnetwork is confined to a chain structure containing 5 lining up nodes. The degree of nodes in this structure is either 2 or 1 . Apparently, local disconnected network is disconnected since the subnetworks are isolated from each other. Consequently, the global shortest path of the network is infinity. On the contrary, the local shortest path is small, 4 in the experiment.

Local connected network is to some extent based on local disconnected network. However, it is no longer disconnected because key linking among the isolated subnetworks emerges to make sure that each node is accessible all over the ring structure of the whole network. Thus the data can also 
flow along the linking to other subnetworks. The APL is computable as 5.26 .

The small-world network in the experiments is an instance of the Watts-Strogatz model [5], which is a random graph generation model that produces graphs with smallworld properties, including short average path lengths and high clustering coefficient. The WS small-world network stems from a regular ring lattice, a graph with 20 nodes, each connected to given number of neighbors, 4 in the experiments. Every node will rewire its edge one by one with a given probability of 0.2 , until rewiring is done by replacing a nonexisting edge. The APL is 2.41 in the experiment, which would change according to the specific structure.

3.2. Group Size. We simulate different size markets by manipulating the amount $(10,20, \ldots, 100)$ of nodes in the network. Then we observe each network's UGR when the equilibrium status is reached. Our interests are as follows. Firstly, whether the UGR is affected by the ascending total of nodes or not. Secondly, whether the UGR is similar under the same group size or not.

3.3. Endowments. The endowments considered here focus on each node's stocks of $g_{1}$ and $g_{2}$. Further study shows that the case can be broken down in detail as follows:

(1) considering $g_{1}$ or $g_{2}$ separately as Index 1 (I1),

(2) considering the sum of $g_{1}$ and $g_{2}$ as Index 2 (I2),

(3) considering the ratio of $g_{1}$ to $g_{2}$ as Index 3 (I3),

(4) considering the total network stocks of $g_{1}$ and $g_{2}$ as Index 4 (I4).

We emphasize how the level (high or low) of these 4 indexes can result in the UGR. The experimental method to examine the 1st, 2nd, and 4th index is modifying the amounts of $g_{1}$ and $g_{2}$; to examine the 3 rd index we check the increase or decrease in UGR before and after removing each node in the network in sequence. Since the initial endowment of goods must be integeral and be randomly assigned, it results in that the amount of goods can hardly take a linear increment. Accordingly, we run the experiments repeatedly and summarize the results to reach the conclusion.

3.4. Trading Sequence. We have mentioned the trade procedure in the economy model, which is a questing node asking for a transaction opportunity with its potential partners in sequence. Obviously, if two or more partners respond to the quest, there would be a queue of nodes waiting for the transaction initiated by the same node. Hence we manage the trading sequence to observe whether the sequence of the queue would have impact on the UGR.

3.5. Turnover and Number of Trades. As UGR, the turnover and number of trades are also important system indicators revealing the activeness of an economy system. We want to find whether these 3 indicators would interact with each other or not.

\section{Experiment Results}

The experiment result summarizes the observation of the factors that may possibly influence the UGR in the simulations.

4.1. Network Structures. We analyze the dynamic behavior of different systems based on global, local disconnected, local connected, and small-world network structures and then compare the UGR, trade times, and turnover of them.

In each regime, trade moves along the edges among the individual agents. In the global network, every agent negotiates and trades with the hub. The global equilibrium is reached quickly. Specifically, the average UGR is $0.3 \%$ at the trade time of 7 and the turnover of 17.238. The hub plays an essential role in keeping this system working, and the other nodes have the same priority to confer with the hub. This structure, however, lays significant dependence on the hub. Although the searching time for trading partners is reduced, opportunities for beneficial exchange among nodes that go wanting may be neglected.

In the second system, local disconnected network, after 37 trades, reaches the equilibrium. The UGR and trade volume drastically rise to $6.9 \%$ and 135.850 , respectively. In the local disconnected network, the nodes are allocated to isolated and equal size groups. Compared with the centralization of global network, local disconnected network is much more like a set of scattering markets. Every group is an independent subsystem trading in accordance with the node index but without transactions with other groups. A local market will not search trade opportunities in other markets after its equilibrium is reached.

The structure of the third system, local connected network, is similar to the local disconnected network. There is local trade, and external trade, carried out with members belonging to other trade groups. Eventually, goods can flow around the entire economy and quickly assimilate global resource constraints after successive trades. To initiate trade in this model, an agent is selected from one group and trades as in local disconnected network, though search excludes individuals outside his group. After every local market reaches its equilibrium, it begins to search transactions from other markets. If a chance was found, the transaction may change the commodity quantities the involving agents possess. Thus, the equilibrium of the corresponding local market may be broken and new opportunities to initiate trades may occur which can improve the UGR. Since the local trade part of this system is the same as the trade in local disconnected network, it can be deduced that as long as the external trade exists, the UGR of local connected network is surely larger than the one of local disconnected network. Furthermore, it can be also inferred that the agents searching for external trade are the keys to increase the UGR of the system. The simulation shows that the UGR in this system rises to $8.6 \%$ and the trade volume and trade times go up to 180.700 and 48 , respectively.

The small-world network is based on the local connected network. Local trades go first in the sense that agents negotiate with subgroup members exclusively before local equilibrium is reached. Compared with the local connected network, 
TABLE 1: The UGR, trade times, and turnover of different structure systems.

\begin{tabular}{lccc}
\hline & UGR (\%) & Trade times & Turnover \\
\hline Global network & 0.3 & 7 & 17.238 \\
Local disconnected network & 6.9 & 37 & 135.850 \\
Local connected network & 8.6 & 48 & 180.700 \\
Small-world network & 10.1 & 53 & 202.539 \\
\hline
\end{tabular}

TABLE 2: Networks statistical characteristics and their UGR.

\begin{tabular}{lccc}
\hline & $\begin{array}{c}\text { APL } \\
\text { (cost) }\end{array}$ & $\begin{array}{c}\text { Clustering } \\
\text { coefficient }\end{array}$ & $\begin{array}{c}\text { UGR } \\
(\%)\end{array}$ \\
\hline Global network & 1.900 & 0 & 0.3 \\
Local disconnected network & $\backslash$ & 0 & 6.9 \\
Local connected network & 5.260 & 0 & 8.6 \\
Small-world network & 2.410 & 0.372 & 10.1 \\
Regular network & 5.000 & 0.500 & 10.7 \\
\hline
\end{tabular}

every agent in the small-world network has probability to develop new edges linking with other unattached agents; namely, every agent may have external transaction. Again, with successive trades, goods can be distributed globally. The equilibrium results for small-world network are as below. The UGR of the small-world network goes up to $10.1 \%$, the trade volume is 202.539 , and trade time are 53. In the small-world network, every agent trades with a fragment of the entire population scattering in any subgroup, thus local equilibrium would be broken more frequently since the market is becoming global. It can be inferred that just a few crosstraders have a remarkable impact on the performance of the system. Table 1 summarizes the results.

Table 2 presents a comparison of the four network structures' key structure characteristics and their UGR. Since the clustering coefficients of global network, local disconnected network, and local connected network are 0 , the regular network with a comparatively high clustering coefficient is taken as a reference.

It appears that although the network structure may exert influence on UGR, the mechanism is a complicated process involving every node and edge. Table 2 reveals that UGR is trying to get a balance between APL and clustering coefficient. The preference of comparatively low cost and high clustering coefficient is reasonable for the extremely low APL which may miss some profit trades but the extremely high clustering coefficient may raise a very high cost.

Degree is a characteristic always being studied based on individuals. So we also raised some node oriented research towards the issues' presumable influence. There are some nodes in the network such as the hub of the global network possessing a degree in sharp contrast with other nodes'. However, despite the high degree of the hub, it usually does not get a higher node UGR than the common nodes, but surely a higher turnover and number of trades. Here node UGR is the consideration of a single node's utility growth rate after the system's equilibrium. It appears that the consideration of a single node's degree counts little for UGR.
TABLE 3: Different size networks and their UGR.

(a) Experimental data of the first 30 rounds simulation

\begin{tabular}{lcccccccccc}
\hline Nodes & 10 & 20 & 30 & 40 & 50 & 60 & 70 & 80 & 90 & 100 \\
\hline UGR $_{\text {avg }}(\%)$ & 5.4 & 3.4 & 11.6 & 7.4 & 7.9 & 22.5 & 9.2 & 10.4 & 5.8 & 14.2 \\
\hline
\end{tabular}

(b) Experimental data of the second 30 rounds simulation

\begin{tabular}{lcccccccccc}
\hline Nodes & 10 & 20 & 30 & 40 & 50 & 60 & 70 & 80 & 90 & 100 \\
\hline UGR $_{\text {avg }}(\%)$ & 12.2 & 6.3 & 8.1 & 4.3 & 13.3 & 5.8 & 12.8 & 2.6 & 21.7 & 8.2 \\
\hline
\end{tabular}

(c) Experimental data of the third 30 rounds simulation

\begin{tabular}{lcccccccccc}
\hline Nodes & 10 & 20 & 30 & 40 & 50 & 60 & 70 & 80 & 90 & 100 \\
\hline UGR $_{\text {avg }}(\%)$ & 3.2 & 8.3 & 3.1 & 4.7 & 6.2 & 7.7 & 5.2 & 9.5 & 10.14 & 11.08 \\
\hline
\end{tabular}

Through these experiments, we find that the network structure would have an impact on UGR, and small-world structure, relying on its low cost and high clustering coefficient gets a highest UGR.

4.2. Group Size. Group size reflects the scale of a market. Empirically, bigger market is more beneficial than a small one in terms of transaction opportunities and profit. The experiment shows that the deduction is not always true. The network structure applied here and in the rest of the paper is small-world network, the one that reaches the highest UGR in the experiments above. The networks are created by WattsStrogaz small-world network generating algorithm, containing $10,20, \ldots, 100$ nodes, respectively. Table 3 , composed of 3 subtables, presents the UGR of different size networks. Each subtable summarizes the group size and its corresponding average UGR $\left(\mathrm{UGR}_{\mathrm{avg}}\right)$ within 30 rounds simulation.

It is obvious that the pattern is disorderly and unsystematic. Firstly, compared to the ascending total of nodes, the UGR is neither ascending nor descending or confirm to a liner function. Secondly, under the circumstance of same amount of nodes, the pattern of UGR is still irregular.

4.3. Endowments. For a clear illustration, we would display an instance of the experiment data (in Table 4) when the economy model runs on the small-world network in Figure 2. In this instance, the network has 20 nodes with the total wealth of 225 and UGR of $14.2 \%$. We pick out node 1 with $5 g_{1}, 3 g_{2}$ (represented as $\mathrm{N1}(5,3)$ ) and node 4 with $8 g_{1}$, $1 g_{2}$ (represented as N6(4,8)) to manipulate their stocks according to the 4 cases (I1, ., I4) listed in Section 3. To keep the global equilibrium of stocks of $g_{1}$ and $g_{2}$, once one node increases or decreases its $g_{1}$ or $g_{2}$, another node has to decrease or increase by the corresponding amount. The modifications of I1, I2, and I3 have to keep to this rule.

The experiments reveal that the separate consideration of $g_{1}$ and $g_{2}$ (Index 1) does not make significant difference to the UGR. Any separate modification on $g_{1}$ or $g_{2}$, may raise, deduct, or maintain the UGR, which is not statistically significant. Thus we deduce that modifying $g_{1}$ or $g_{2}$ separately does not much affect the UGR. The experiments also confirm that an increase or decrease of a node's $g_{1}$ and $g_{2}$ simultaneously (Index 2) can improve the network's UGR. 
TABle 4: Modifications to the endowments and their corresponding UGR.

(a) Separate modification of $g_{1}$ (I1)

\begin{tabular}{lccccccc}
\hline N1, N6 & $(1,3),(8,8)$ & $(2,3),(7,8)$ & $(3,3),(6,8)$ & $(4,3),(5,8)$ & $(6,3),(3,8)$ & $(7,3),(2,8)$ & $(8,3),(1,8)$ \\
\hline UGR (\%) & 13.9 & 14.3 & 14.2 & 14.2 & 14.0 & 14.2 & 14.2 \\
\hline
\end{tabular}

(b) Modifyingthe sum of $g_{1}$ and $g_{2}$ (I2)

\begin{tabular}{lccc}
\hline N1, N6 & $(6,4),(3,7)$ & $(7,5),(2,6)$ & $(8,6),(1,5)$ \\
\hline UGR (\%) & 14.3 & 14.3 & 14.4 \\
\hline
\end{tabular}

The experiments also reveal that a node with relatively high or low ratio of $g_{1}, g_{2}$ (Index 3 ) plays an import role in a network, that is, deleting these nodes may cause remarkable variation of UGR. However, intentionally appending edges to those nodes cannot definitely lead to an increase of UGR. We also confirm that the correlation between total stock of $g_{1}, g_{2}$ and the global UGR (Index 4) is hard to find. The pattern of the correlation is irregular. More specifically, a high total endowment network may have a similar UGR with low total endowment network, and the same endowment may cause significantly different UGR. Consequently, we consider that the total endowment has little relevance to UGR. Table 4 presents the UGR when the stocks of N1 and N6 are modified according to I1 and I3.

These experiments show that an effective way to improve UGR is to increase or decrease the node's $g_{1}$ and $g_{2}$ simultaneously, that is, to exacerbate the stocking gap among the nodes.

4.4. Trading Sequence. The result is that when the network reaches its equilibrium, whatever the trade sequence is, the UGR would not have any correction. However, if the trade stops before the equilibrium, the UGR would be changed and the node UGR would also diverse from the status of equilibrium.

4.5. Turnover and Number of Trades. Table 1 compares the correlation among UGR, turnover, and the number of trades in four different network structures. Clearly, the turnover is in proportion to the trade rounds and the UGR is always in proportion to them. Sometimes, it happens that the number of trades of local connected and WS small-world network is the same. However, the UGR of the latter is beyond that of the former. It stems from the pairs of the trading nodes are different, which are constrained by the network structure. Thus, we can deduce that the turnover and number of trades are not the direct incentives of UGR, and the underlying base is still the network structure.

\section{Conclusion}

This paper uses economic theory, network theory, and computer simulations to examine the factors which may affect the utility growth atio. Major findings are summarized as follows.

(1) Under the circumstance of same endowments, the network structure is proved to have a notable impact on the UGR. The small-world network has several characteristics that suggest they may be an efficient economic system structure. We think the important attributes of small-world networks play a part in it. More specifically, the low short average path length, defined as the averaged minimum number of trading required for a node to trade with its neighbors, provides a low trade cost, and the high clustering coefficient, indicating a high degree the nodes would like to create tightly knit groups characterized by a relatively high density of ties, provides an environment in which agents have high probability to trade with all the potential partners.

(2) The experiments reveal that an increase or decrease of a node's $g_{1}$ and $g_{2}$ simultaneously can always improve the network's UGR. Meanwhile, deleting the nodes with relatively high or low ratio of $g_{1}$ and $g_{2}$ may cause remarkable variations of UGR. However, intentionally appending edges to those nodes cannot definitely lead to an increase of the UGR. It is also proven that the separate correction of goods 1 and 2 does not make significant difference to the UGR. Moreover, the pattern of the correlation between total stock of $g_{1}, g_{2}$ and the global UGR is irregular.

(3) Although the turnover and number of trades are in direct proportion to the UGR, they are not the direct incentives of UGR. The underlying mechanism is still the network structure. Besides, the trading sequence would not affect UGR unless the trade stops before the equilibrium.

\section{Conflict of Interests}

The authors declare that there is no conflict of interests regarding the publication of this paper.

\section{References}

[1] X. F. Wang, "Complex networks: topology, dynamics and synchronization," International Journal of Bifurcation and Chaos, vol. 12, no. 5, pp. 885-916, 2002.

[2] S.-H. Yook, H. Jeong, and A.-L. Barabási, "Modeling the internet's large-scale topology," Proceedings of the National Academy of Sciences of the United States of America, vol. 99, no. 21, pp. 13382-13386, 2002.

[3] G. Sanjeev, M. J. van der Leij, and J. L. Moraga-Gonzalez, "Economics: an emerging small world?" TI Discussion Paper 
04-001/1, Tinbergen Institute, Rotterdam, The Netherlands, 2004.

[4] H. Hong, M. Y. Choi, and B. J. Kim, "Synchronization on smallworld networks," Physical Review E, vol. 65, no. 2, Article ID 026139, pp. 026139/1-026139/5, 2002.

[5] D. J. Watts and S. H. Strogatz, "Collective dynamics of "smallworld” networks,” Nature, vol. 393, no. 6684, pp. 440-442, 1998.

[6] P. Erdős and A. Rényi, “On random graphs. I," Publicationes Mathematicae Debrecen, vol. 6, pp. 290-297, 1959.

[7] D. J. Watts, Small Worlds, Princeton University Press, Princeton, NJ, USA, 1999.

[8] A. Medina, I. Matta, and J. Byers, "On the origin of power-laws in internet topologies," Computer Communication Review, vol. 2, no. 30, pp. 18-28, 2000.

[9] J. Winick, C. Jin, Q. Chen, and S. Jamin, "Inet: internet topology generator, Tech. Rep. CSE-TR-443-00, Department of EECS, University of Michigan, 2000.

[10] R. Pastor-Satorras and A. Vespignani, "Epidemic spreading in scale-free networks," Physical Review Letters, vol. 86, no. 14, pp. 3200-3203, 2001.

[11] R. Pastor-Satorras and A. Vespignani, "Epidemic dynamics and endemic states in complex networks," Physical Review E, vol. 63, no. 6, pp. 066117/1-066117/8, 2001.

[12] R. F. I. Cancho and R. V. Solé, "The small world of human language," Proceedings of the Royal Society B, vol. 268, no. 1482, pp. 2261-2265, 2001.

[13] M. E. J. Newman, "Scientific collaboration networks. I. Network construction and fundamental results," Physical Review E, vol. 64, no. 1, pp. 016131/1-016131/8, 2001.

[14] M. Batty, "Editorial," Environment and Planning B, vol. 33, no. 5, pp. 637-638, 2006.

[15] J. Wang, H. Mo, F. Wang, and F. Jin, "Exploring the network structure and nodal centrality of China's air transport network: a complex network approach," Journal of Transport Geography, vol. 19, no. 4, pp. 712-721, 2011.

[16] N. Cattan, "Attractivity and internationalisation of major European cities: the example of air traffic," Urban Studies, vol. 32, no. 2, pp. 303-312, 1995.

[17] A. M. Bell, "Bilateral trading on a network: a simulation study," in Proceedings of the Working Notes: Artificial Societies and Computational Markets Autonomous Agents Workshop, St. Paul, Minneapolis, Minn, USA, 1998.

[18] G. Li, H. Yang, L. Sun, P. Ji, and L. Feng, "The evolutionary complexity of complex adaptive supply networks: a simulation and case study," International Journal of Production Economics, vol. 124, no. 2, pp. 310-330, 2010.

[19] A. Wilhite, "Bilateral trade and "small-world" networks," Computational Economics, vol. 18, no. 1, pp. 49-64, 2001.

[20] E. Garfield, "It's a small world after all," Current Contents, vol. 43, pp. 5-10, 1979.

[21] L. Egghe and R. Rousseau, Introduction to Informetrics, Elsevier, Amsterdam, The Netherlands, 1990.

[22] S. Maslov and K. Sneppen, "Specificity and stability in topology of protein networks," Science, vol. 296, no. 5569, pp. 910-913, 2002.

[23] D. W. Caves and L. R. Christensen, "Global properties of flexible functional forms," The American Economic Review, vol. 70, no. 3, pp. 422-432, 1980.

[24] A. Feldman, "Bilateral trading processes, pairwise optimality, and Pareto optimality," Review of Economic Studies, vol. 40, no. 4, pp. 463-473, 1973.
[25] A. Sen, "Markets and freedoms: achievements and limitations of the market mechanism in promoting individual freedoms," Oxford Economic Papers, vol. 45, no. 4, pp. 519-541, 1993.

[26] R. Diestel, Graph Theory, vol. 173, Springer, Berlin, Germany, 3rd edition, 2005.

[27] S. Milgram, “The small-world problem," Psychology Today, vol. 2, pp. 60-67, 1967.

[28] V. Latora and M. Marchiori, "Economic small-world behavior in weighted networks," European Physical Journal B, vol. 32, no. 2, pp. 249-263, 2003.

[29] P. W. Holland and S. Leinhardt, "Transitivity in structural models of small groups," Comparative Group Studies, vol. 2, pp. 107-124, 1971.

[30] A. Kemper, Valuation of Network Effects in Software Markets: A Complex Networks Approach, Springer, Heidelberg, 2010.

[31] M. O. Jackson and A. Wolinsky, "A strategic model of social and economic networks," Journal of Economic Theory, vol. 71, no. 1, pp. 44-74, 1996.

[32] Y. Ioannides, "Evolution of trading structures," in The Economy as an Evolving Complex System II, W. B. Arthur, S. Durlauf, and D. Lane, Eds., Addison-Wesley, 1997. 


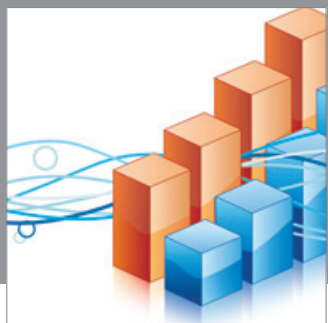

Advances in

Operations Research

mansans

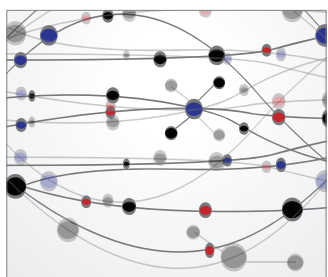

The Scientific World Journal
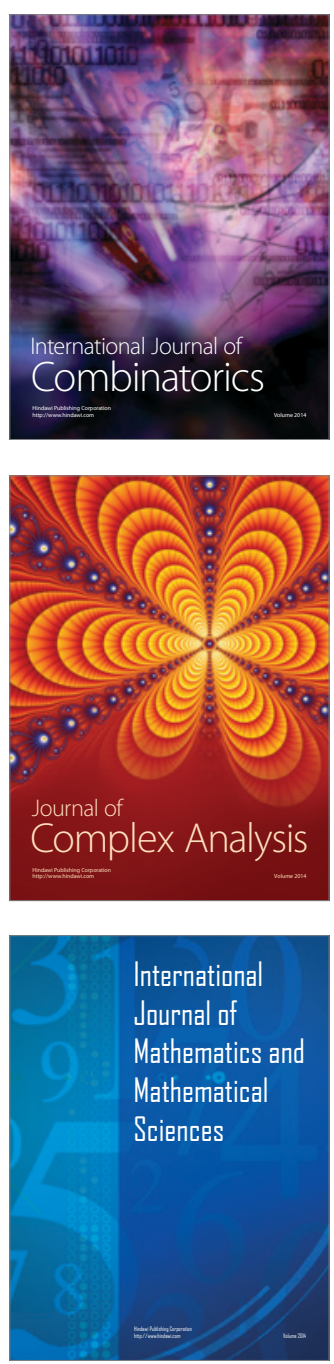
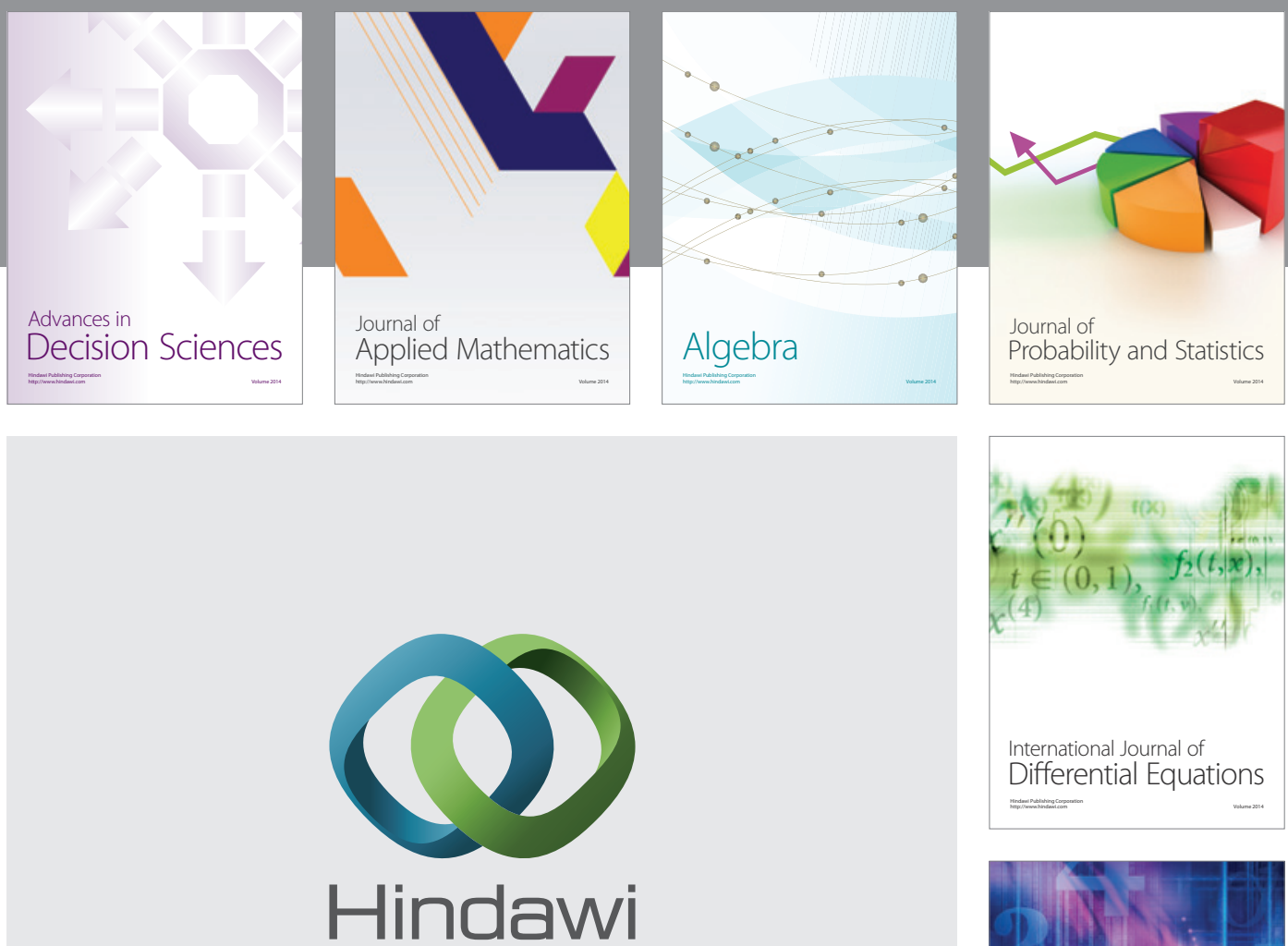

Submit your manuscripts at http://www.hindawi.com
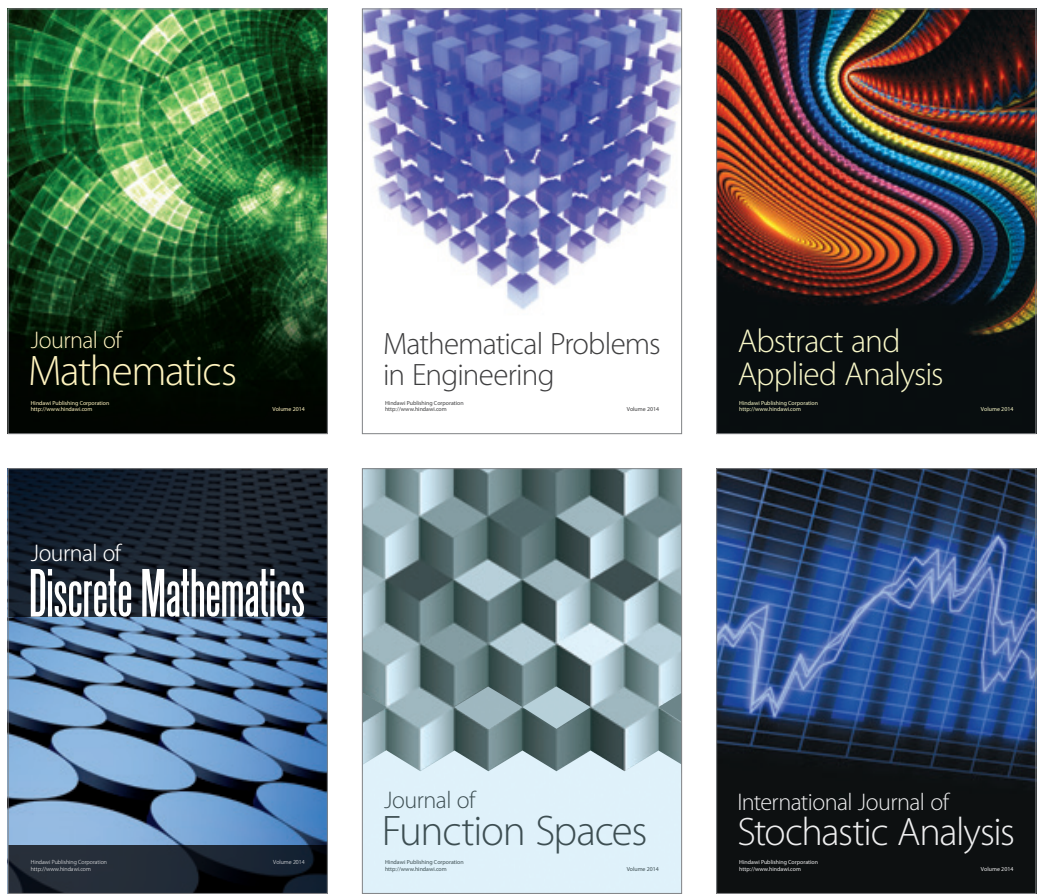

Journal of

Function Spaces

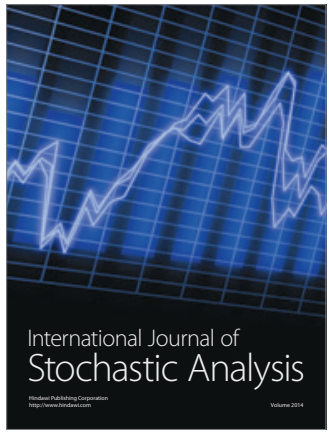

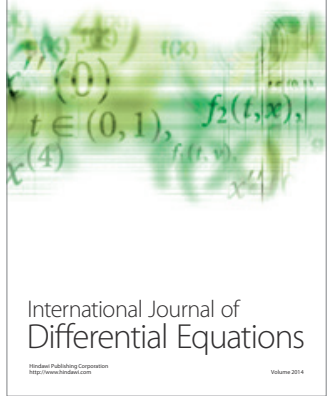
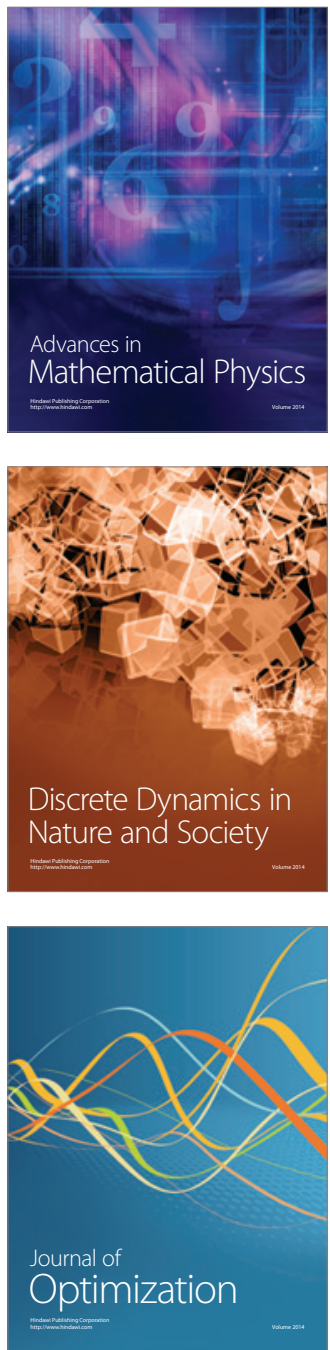